The Euler equations for (6) constitute the complete system of equations of an anisotropic plate. It is noted that the term $P_{i}^{*(n)}$ in the stress equation of motion given by (6) is a function of the surface traction. This automatically takes care of the traction boundary conditions in the interior of the plate, while the integral over $A_{u}$ in (6) yields the displacement boundary conditions in the interior of the plate. In fact, (6) yields in a simple manner all conditions at the edge as well as in the interior of the plate for equations of all orders.

It is noted that the plate equations written from (6) are essentially those given by Mindlin $[4,5]$, who used the ordinary variational equation of motion in deriving the stress equations of motion and Neumann's method in deriving the boundary conditions. Mindlin's procedure is thus also justified from the variational point of view.

There is no difficulty in extending the present treatment of plates to shells. The results are reported elsewhere [6].

\title{
REFERENCES
}

1. Yi-Yuan Yu, Generalized Hamilton's principle and variational equation of motion in nonlinear elasticity theory, with application to plate theory, J. Acoustical Soc. Amer. 36 (1964) 111

2. Hai-Chang $\mathrm{Hu}, \mathrm{On}$ some variational principles in the theory of elasticity and the theory of plasticity, Acta Physica Sinica 10 (1954) 259, also Scientia Sinica 4 (1955) 33

3. K. Washizu, On the variational principles of elasticity and plasticity, M. I. T. Aeroelastic Structures Res. Lab. Tech. Report 25.18 (1955)

4. R. D. Mindlin, An introduction to the mathematical theory of vibrations of elastic plates, U. S. Army Signal Corps, Fort Monmouth, N. J., 1955

5. R. D. Mindlin, High frequency vibrations of crystal plates, Q. Appl. Math. 19 (1961) 51

6. Yi-Yuan Yu, On linear equations of isotropic elastic plates and shells, Scientific Report No. 2, Polytechnic Institute of Brooklyn, Dept. of Mech. Engg., 1964

\section{A CORRESPONDENCE PRINCIPLE FOR VISCOELASTIC WAVE PROPAGATION*}

By I. HERRERA, (University of Mexico) AND M. E. GURTIN, (Brown University)

Introduction. It is a well known result of classical linear elasticity theory that the speed of propagation $U$ of an acceleration wave, at a point $x$ in an elastic solid of elasticity tensor $c_{i j k l}(x)$ and density $\rho(x)$, is a solution to the eigenvalue problem**

$$
\left[c_{i j k l}(x) n_{i} n_{l}-\rho(x) U^{2} \delta_{i k}\right] a_{k}=0 .
$$

Here the unit vector $n$ is the direction of propagation at $x$. Since the elasticity tensor obeys the symmetry relations

$$
c_{i j k l}=c_{k l i i},
$$

it is clear that (1.1) has three (not necessarily distinct) solutions $U_{1}, U_{2}, U_{3}$. More-

*Received March 13, 1964.

**We use indicial notation and Cartesian tensors throughout. Thus subscripts have the range $(1,2,3), x_{i}$ denote rectangular Cartesian coordinates, and $x=\left(x_{1}, x_{2}, x_{3}\right)$. Moreover summation over repeated subscripts is implied, $\delta_{i j}$ is Kronecker's delta, subscripts preceded by a comma indicate differentiation with respect to the corresponding Cartesian coordinate. 
over these solutions will be real and nonzero if and only if $c_{i j k l}(x)$ is strongly elliptic in the sense that

$$
c_{i j k l}(x) n_{j} n_{l} v_{i} v_{k}>0 \text { whenever } v \neq 0 .
$$

A recent result of Toupin and Gurtin [1], which is a generalization to anisotropic materials of a theorem due to Gurtin and Sternberg [2], demonstrates the connection between wave propagation and uniqueness. This result may be stated roughly as follows: the displacement problem of linear elastodynamics has at most one solution provided $c_{i j k l}$ is constant and strongly elliptic. Thus, if the medium is homogeneous, a sufficient condition for uniqueness is that three linearly independent acceleration waves can propagate in every direction.

The foregoing results can be easily generalized to visco-elastic solids with the aid of a correspondence principle which we prove in this paper. This principle asserts that the speed of propagation $U$ of an acceleration wave at a point $x$ in a viscoelastic solid with relaxation tensor $G_{i j k l}(x, t)$ and density $\rho(x)$ satisfies

$$
\left(G_{i j k l}(x, 0) n_{j} n_{l}-\rho(x) U^{2} \delta_{i k}\right) a_{k}=0 .
$$

Hence, by (1.1), this speed is identical to the speed of propagation through an elastic solid whose elasticity tensor is

$$
c_{i j k l}(x)=G_{i j k l}(x, 0) .
$$

This correspondence principle brings out the remarkable (but intuitively obvious) fact that the speed of propagation of an acceleration wave through a viscoelastic solid depends only upon the initial value $G_{i j k l}(x, 0)$ of the relaxation tensor and is independent of the behavior of $G_{i j k l}(x, t)$ with $t$.

Assume for the remainder of this section that $G_{i j k l}(x, t)$ is initially symmetric, i.e.,

$$
G_{i j k l}(x, 0)=G_{k l i j}(x, 0) .
$$

Then the eigenvalue problem (1.4) has three solutions. Further these solutions will be real and non-zero if and only if $G_{i j k l}(x, t)$ is initially strongly elliptic, that is if and only if $G_{i j k l}(x, 0)$ is strongly elliptic.

Edelstein and Gurtin [3] have shown that the displacement problem of dynamic viscoelasticity theory has at most one solution provided $G_{i j k l}(x, t)$ is independent of $x$ and initially strongly elliptic. Thus a sufficient condition for uniqueness to hold in a homogeneous viscoelastic medium is that three independent acceleration waves can propagate in every direction in the body.

The correspondence principle. The fundamental system of field equations in the linear theory of viscoelasticity consists of the following:**

$$
\begin{gathered}
2 \epsilon_{i j}(x, t)=u_{i, j}(x, t)+u_{j, i}(x, t), \\
\sigma_{i j, j}(x, t)+f_{i}(x, t)=\rho(x) u_{i}^{(2)}(x, t), \quad \sigma_{i j}(x, t)=\sigma_{i i}(x, t), \\
\sigma_{i j}(x, t)=\int_{-\infty}^{t} G_{i j k l}(x, t-s) \epsilon_{k l}^{(1)}(x, s) d s .
\end{gathered}
$$

*Notice that $G_{i j k l}(x, 0)$ automatically has this property if it is isotropic.

${ }^{* *}$ We use the notation $g^{(n)}=\partial^{n} g / \partial t^{n}$. 
Here $u_{i}(x, t), \epsilon_{i j}(x, t), \sigma_{i j}(x, t)$, and $f_{i}(x, t)$ are the Cartesian components of the displacement vector, the strain tensor, the stress tensor, and the body force density vector defined for every pair $(x, t)$ such that $x$ is a point of the open region $R$ occupied by the interior of the body and $t(-\infty<t<\infty)$ is the time. Further $\rho(x)$ is the mass density defined for every $x \varepsilon R$, and $G_{i j k l}(x, t)$ are the components of the relaxation tensor which are defined for every $(x, t) \varepsilon R \times[0, \infty)$ and satisfy

$$
G_{i j k l}=G_{i i k l}=G_{i j l k} \text {. }
$$

The first symmetry relation follows from the symmetry of the stress tensor, while the second follows (without loss in generality) from the symmetry of the strain tensor.

We suppose once and for all that we are given a solution $u_{i}, \epsilon_{i j}, \sigma_{i i}$ of (2.1), (2.2), (2.3) on $R \times(-\infty, \infty)$ which meets the initial condition

$$
u_{i}(x, t)=0, \quad(x, t) \varepsilon \Re \times(-\infty, 0)
$$

and corresponds to data $\rho, G_{i j k l}, f_{i}$ which satisfies:

(i) $\rho>0$ is continuous;

(ii) $G_{i j k l}$ is twice continuously differentiable and meets (2.4);

(iii) $f_{i}$ is continuous.

Moreover we assume the existence of an acceleration wave propagating through $R$-that is a one-parameter family of surfaces $S_{t}(0 \leq t<\infty)$ which has the following properties:

(iv) the hypersurface

$$
\sum_{s}=\left\{(x, t) \mid x \varepsilon S_{t}(0 \leq t<\infty)\right\}
$$

is smooth and orientable;

(v) given an $x \varepsilon R$, the set

$$
D_{x}=\left\{t \mid x \varepsilon S_{t}\right\}
$$

has measure zero;

(vi) $u_{i}, u_{i}^{(1)}, u_{i, j}$ are continuous on $R \times(-\infty, \infty), u_{i}^{(2)}, u_{i, i}^{(1)}, u_{i, j k}$ have jump discontinuities across $\sum_{s}$ but are continuous everywhere else on $R \times(-\infty, \infty)$, and $u_{i, j k}$ is bounded on compact subsets of $R \times(-\infty, \infty)$.

Let $(x, t) \varepsilon \sum_{s}$. Condition (iv) then implies the existence of a normal vector $\nu$ at $(x, t)$. Indeed, such a vector is given by

$$
\nu=\left(n_{1}, n_{2}, n_{3},-U\right),
$$

where $n_{i}$ are the components of a unit vector which is normal to $S_{t}$ at $x$ and $U \geq 0$. The latter condition is imposed to insure the uniqueness of $\nu$ at points where $U \neq 0$. The number $U$ is called the speed of propagation of $S_{t}$ at $x$, while the unit vector $n$ is called the direction of $S_{t}$ at $x$. Condition $(v)$ is merely the requirement that the surface $S_{t}$, as it progresses in time, does not pass through a given point $x$ too many times. This condition together with condition (vi) implies that for any $x \varepsilon R, u_{i, j k}(x, t)$, $u_{i, i}^{(1)}(x, t)$, and $u_{i}^{(2)}(x, t)$ are discontinuous only when $t \varepsilon D_{x}$. Finally we remark that all integrals are to be taken in the sense of Lebesgue.

We are now in a position to prove the Correspondence Principle for Wave Propagation. The speed of propagation $U$ of the acceleration wave $S_{t}$ at a point $x \varepsilon S_{t}$ is a solution of the eigenvalue problem

$$
\left(c_{i j k l} n_{j} n_{l}-\rho U^{2} \delta_{i k}\right) a_{k}=0,
$$


where

$$
c_{i j k l}=G_{i j k l}(x, 0)
$$

and $n$ is the direction of $S_{\imath}$ at $x$. That is, the speed of propagation is identical to the speed of propagation of $S_{t}$ through an elastic solid whose elasticity tensor is $c_{i j k l}$.

Proof. Integrate (2.3) by parts and use (2.1), (2.5) to verify that

$$
\sigma_{i j}=c_{i j k l} \epsilon_{k l}+G_{i j k l}^{(1)} * \epsilon_{k l},
$$

where $c_{i j k l}$ is $\mathrm{g} \cdot$ ven by $(2.8)$ and

$$
G_{i j k l}^{(1)} * \epsilon_{k l}(x, t)=\int_{-\infty}^{t} G_{i j k l}^{(1)}(x, t-s) \epsilon_{k l}(x, s) d s .
$$

Now substitute (2.1), (2.9) into (2.2) and use the symmetries (2.4) together with (2.8) to derive the displacement equations of equilibrium in the form

$$
c_{i j k l} u_{k, l i}=\rho u_{i}^{(2)}-F_{i},
$$

where

$$
F_{i}=c_{i j k l, j} u_{k, l}+G_{i j k l, i}^{(1)} * u_{k, l}+G_{i j k l}^{(1)} * u_{k, l i}+f_{i} .
$$

Define the functions $p_{k l}$ and $q_{i}$ through

$$
p_{k l}=u_{k, l}, \quad q_{i}=u_{i}^{(1)} .
$$

Then $p_{k l}$ and $q_{i}$ are continuous, have continuous derivatives except on $\sum_{s}$, and satisfy the compatibility conditions

$$
p_{k l}^{(1)}=q_{k, l} .
$$

Moreover, the jumps in the (four dimensional) gradients of $p_{k l}$ and $q_{i}$ are parallel to the normal $\nu$ of $\sum_{s}$ and hence, because of (2.6), take the form*

$$
\begin{aligned}
{\left[p_{k l, j}\right] } & =\lambda_{k l} n_{j}, \\
{\left[p_{k l}^{(1)}\right] } & =-\lambda_{k l} U, \\
{\left[q_{k, l}\right] } & =a_{k} n_{l}, \\
{\left[q_{k}^{(1)}\right] } & =-a_{k} U .
\end{aligned}
$$

Thus (2.13), (2.14), (2.15), together with the continuity of $c_{i i k l}$ and $\rho$ across $\sum_{s}$ imply

$$
\left[c_{i j k l} u_{k, l j}\right]=c_{i j k l} \lambda_{k l} n_{i}, \quad\left[\rho u_{i}^{(2)}\right]=-\rho a_{i} U, \quad-\lambda_{k l} U=a_{k} n_{l} .
$$

By (2.8) and the smoothness assumptions (ii), (iii), and (vi) it follows that

$$
\left[c_{i j k l, j} u_{k, l}\right]=\left[G_{i j k l, i}^{(1)} * u_{k, l}\right]=\left[f_{i}\right]=0 .
$$

We now prove that $\left[G_{i j k l}^{(1)} * u_{k, l i}\right]=0$. Fix $(x, t) \varepsilon \sum_{s}$, let $A=G_{i j k l}^{(1)} * u_{k, l i}$, and notice that

${ }^{*}$ Here and in the sequel we use the usual notation for the jump $[g]$ of a function $g$ across $\Sigma{ }_{\text {s. }}$ That is

$$
[g](x, t)=\lim _{\left(x^{+}, t^{+}\right) \rightarrow(x, t)} g\left(x^{+}, t^{+}\right)-\lim _{\left(x^{-}, t^{-}\right) \rightarrow(x, t)} g\left(x^{-}, t^{-}\right),
$$

where $\left(x^{+}, t^{+}\right)$and $\left(x^{-}, t\right)$ lie on opposite sides of $\Sigma_{s}$. 
$A\left(x^{+}, t^{+}\right)=\int_{0}^{t} G_{i j k l}^{(1)}\left(x^{+}, t^{+}-s\right) u_{k, l i}\left(x^{+}, s\right) d s+\int_{t}^{t^{+}} G_{i j k l}^{(1)}\left(x^{+}, t^{+}-s\right) u_{k, l i}\left(x^{+}, s\right) d s$.

Let $\Lambda$ denote the compact set $\Omega \times[0, T]$, where $\Omega$ is a solid sphere centered at $x$ and contained in $R$ while $T>t$. Then hypotheses (ii) and (vi) imply that $G_{i i k l}^{(1)}$ and $u_{k, l i}$ are bounded on $\Lambda$. Thus the second term in (2.18) tends to zero as $\left(x^{+}, t^{+}\right) \rightarrow(x, t)$. Moreover conditions $(i i),(v)$, and $(v i)$ yield

$$
G_{i j k l}^{(1)}\left(x^{+}, t^{+}-s\right) u_{k, l i}\left(x^{+}, s\right) \rightarrow G_{i j k l}^{(1)}(x, t-s) u_{k, l j}(x, s)
$$

for almost every $s \leq t$, i.e., for $s \notin D_{x}$. Therefore the boundedness of $G_{i j k l}^{(1)}$ and $u_{k, l i}$ on $\Lambda$ and Lebesgue's bounded convergence theorem imply

$$
\lim _{\left(x^{+}, t^{+}\right) \rightarrow(x, t)} A\left(x^{+}, t^{+}\right)=\int_{0}^{t} G_{i j k l}^{(1)}(x, t-s) u_{k, l j}(x, s) d s .
$$

Clearly the same result follows for $\lim _{\left(x^{-}, t^{-}\right) \rightarrow(x, t)} A\left(x^{-}, t^{-}\right)$and thus

$$
\left[G_{i j k l}^{(1)} * u_{k, l i}\right]=0 .
$$

Equations (2.12), (2.17), and (2.22) imply $\left[F_{i}\right]=0$. It therefore follows from (2.11), (2.16) that

$$
\left(c_{i j k l} n_{j} n_{l}-\rho U^{2} \delta_{i k}\right) a_{k}=0,
$$

and this completes the proof.

Acknowledgement. The authors are indebted to W. Edelstein who read the manuscript and offered many helpful criticisms. This research was supported in part (M. E. G.) by the Office of Naval Research under Contract Nonr 562(25) and in part (I. H) by the Institutes of Geophysics and Engineering at the University of Mexico.

\section{REFERENCES}

1. R. A. Toupin and M. E. Gurtin, $A$ uniqueness theorem for the displacement boundary-value problem of linear elastodynamics, to appear in Q. Appl. Math.

2. M. E. Gurtin and Eli Sternberg, A note on uniqueness in classical elastodynamics, Q. Appl. Math., 19 (1961) 169-171

3. W. S. Edelstein and M. E. Gurtin, Uniqueness theorems in the linear theory of anisotropic viscoelastic solids, Rept. No. 24, Contract Nonr 562 (25), Brown University, February, 1964.

\section{IRREVERSIBLE THERMODYNAMICS OF PERFECT PLASTICITY*}

By N. MORGENSTERN, (Imperial College of Science \& Technology, London, England)

1. Introduction. For the solution of boundary value problems in continuum mechanics, it is necessary to supplement the equation of continuity that expresses the conservation of mass and the equation of motion with the constitutive equation for the material under consideration. In the case of plasticity, the constitutive equation introduces the mechanical properties of the material by means of the relation between the

*Received October 11, 1963; revised manuscript received February 20, 1964. 